\title{
The modest impact of transcription factor Nrf2 on the course of disease in an ALS animal model
}

\author{
Yansu Guo 1,2,3, Yuesheng Zhang ${ }^{4}$, Di Wen ${ }^{1}$, Weisong Duan ${ }^{1,2,3}$, Ting An ${ }^{1}$, Pengxiao Shi ${ }^{1}$, Jingjing Wang ${ }^{1}$, \\ Zhongyao $\mathrm{Li}^{1,2,3}$, Xiaoyu Chen ${ }^{1}$ and Chunyan $\mathrm{Li}^{1,2,3}$
}

Oxidative stress is associated with the pathogenesis of amyotrophic lateral sclerosis (ALS). Nuclear factor erythroid 2-related factor 2 (Nrf2)-antioxidant response element (ARE) pathway is one of the major cellular defense mechanisms against oxidative stress. However, the role of Nrf2-mediated neuroprotection (antioxidant defense) in the disease development of ALS remains unclear. To further investigate the role of Nrf2 in ALS, we genetically eliminate the Nrf2 gene from SOD1-G93A mice, a commonly used ALS mouse model, by generating a double mutant (Nrf2 - / - SOD1-G93A mice). We found that it only had a modest impact on the course of disease by knocking out Nrf2 gene in these mice. Further studies demonstrated that, among previously known Nrf2-regulated phase II enzymes, only $\mathrm{NAD}(\mathrm{P}) \mathrm{H}$ : quinone oxidoreductase 1 induction was significantly affected by the elimination of Nrf2 gene in SOD1-G93A mice. Taken together, our data suggested that Nrf2 is not the sole mediator for the induction of antioxidant genes in SOD1-G93A mice, and Nrf2-mediated neuroprotection is not the key protective mechanism against neurodegeneration in those mice.

Laboratory Investigation (2013) 93, 825-833; doi:10.1038/labinvest.2013.73; published online 27 May 2013

KEYWORDS: amyotrophic lateral sclerosis (ALS); cytoprotective phase 2 enzyme; NF-E2-related factor 2 (Nrf2); oxidative stress; SOD1

Amyotrophic lateral sclerosis (ALS) is a progressively debilitating neurodegenerative disease, characterized by upper and lower motor neuron loss and eventually results in paralysis and death. ${ }^{1}$ SOD1 mutations contribute to $20 \%$ of familial ALS, which is $\sim 5-10 \%$ of total ALS cases. ${ }^{2}$ SOD1-G93A transgenic mice replicate most phenotypes of human ALS and are used widely in the pathogenetic and therapeutic studies. ${ }^{3}$ It is well accepted that Oxidative stress contributes to the pathogenesis of ALS. ${ }^{4-6}$ Nuclear factor erythroid 2related factor 2 (Nrf2), a member of the 'cap ' $n$ ' collar' family of basic leucine zipper transcription factors, ${ }^{7}$ binds to antioxidant response element (ARE) in the upstream region of its target genes promoting transcription of various cytoprotective genes, such as heme oxygenase-1 (HO- 1$),{ }^{8}$ $\mathrm{NAD}(\mathrm{P}) \mathrm{H}$ : quinone oxidoreductase 1 (NQO1), ${ }^{9}$ and the catalytic subunit of glutamate cysteine ligase (GCLC), ${ }^{10}$ which helps restore the oxidant-antioxidant balance in cells. ${ }^{10,11}$ In this context, several studies demonstrated that Nrf2/ARE activators provided robust neuroprotective effect and ameliorated the disease progression in SOD1-G93A transgenic mice. ${ }^{12,13}$ Notably, upregulation of $\mathrm{Nrf2}$ expression was found in the nucleus of motor neurons in lumbar anterior horn of ALS model mice. ${ }^{14}$ We also reported that significant HO-1 induction was detected in the lumbar spinal cord of ALS mice. ${ }^{15}$ Overall, those findings support the notion that Nrf2 pathway-mediated neuroprotection might be important in protecting neurons against the insults of oxidative stress during the disease development of ALS.

To further evaluate the potential role of Nrf2 pathway played in the neurodegenerative process in ALS, we knocked out the Nrf2 gene from SOD1-G93A mice by crossbreeding Nrf2-null mice with SOD1-G93A mice. Surprisingly, only modest deterioration on disease onset and life span was observed in Nrf2-/-SOD1-G93A mice compared with $\mathrm{Nrf2}+/+$ SOD1-G93A animal. The disease onset age was advanced by $7.5-10.5$ days and the life span was shortened by 5.5-7.1 days in these double mutant animals ( $\mathrm{Nrf2}-/-$ SOD1-G93A). We found that only NQO1 induction was disabled in the target tissues and astrocytes of Nrf2 - / SOD1-G93A mice, whereas the expression of HO-1 and GCL remains unchanged in these animals. In addition, the tissue level of GSH (glutathione), a major cellular antioxidant, was comparable between Nrf2 - / - SOD1-G93A mice and Nrf2 + / + SOD1-G93A animals. Overall, our data suggested

\footnotetext{
${ }^{1}$ Department of Neurology, the Second Hospital of Hebei Medical University, Shijiazhuang, China; ${ }^{2}$ Key Laboratory of Hebei Neurology, Shijiazhuang, China; ${ }^{3}$ Hebei Institute of Cardiocerebrovascular Disease, Shijiazhuang, China and ${ }^{4}$ Department of Chemoprevention, Roswell Park Cancer Institute, Buffalo, NY, USA Correspondence: Professor C Li, MBBS, Department of Neurology, the Second Hospital of Hebei Medical University, 215 Heping Xi Road, Shijiazhuang 050000, China. E-mail: chunyanli5@yahoo.com.cn 
that Nrf2 has a limited role in preventing disease development and facilitating antioxidant defense in SOD1-G93A transgenic mice.

\section{MATERIALS AND METHODS \\ Mice}

The generation and genotyping of SOD1-G93A mice had been described previously. ${ }^{3}$ Wild-type $(\mathrm{Nrf} 2+/+)$ and Nrf2-deficient (Nrf2-/-) CD1/ICR mice were obtained from Dr Thomas W. Kensler (Johns Hopkins University, Baltimore, MD) and were genotyped using primers as follows: NRF5 (in Nrf2 gene), 5'-TGGACGGGACTATTG AAGGCTG-3'; NAS (in Nrf2 gene), $5^{\prime}$-GCCGCCT TTTCAGTAGATGGAGG-3'; and NLACZ (in LacZ gene), 5'-GCGGATTGACCGTAATGGGATAGG-3' . SOD1-G93A/Nrf2 $+/+$ and SOD1-G93A/Nrf2 - / - mice were generated by mating SOD1-G93A transgenic male mice with $\mathrm{Nrf} 2+/+$ and $\mathrm{Nrf2}-/$ - female mice, respectively, for 10 generations. The animals were housed in a temperature-, light-, and humidity-controlled environment and fed at libitum and drank water freely.

\section{Animal Experiments}

To monitor the disease progression, all animals were inspected daily for signs of motor deficit and weighed twice per week, starting at 12 weeks of age. Disease onset was determined when the following criteria were both met: (1) two continuous weight losses were observed after the animals reached their peak body weight; (2) earliest gait abnormalities were shown. The end stage was defined as the animals can no longer right themselves $30 \mathrm{~s}$ after being placed on their backs or sides. ${ }^{16}$ The animals were killed at various time points (pre-symptomatic stage, disease onset, and end stage), and the tissues including spinal cord and cortex were collected thereafter. Animal experiments were carried out according to the regulations of laboratory animal management promulgated by the Ministry of Science and Technology of the People's Republic of China, which are in accordance with the guidelines published in the NIH Guide for the Care and Use of Laboratory Animals.

\section{Primary Astrocyte Culture}

Primary astrocyte culture was established from brain cortex tissue of 1-day-old $\mathrm{Nrf} 2+/+$ or $\mathrm{Nrf} 2-/-$ mice, as described. ${ }^{17}$ The dissociated astrocytes were kept in glial medium containing DMEM (Invitrogen) supplemented with $10 \%$ FBS, $100 \mathrm{U} / \mathrm{ml}$ penicillin (Amrisco), and $100 \mathrm{mg} / \mathrm{ml}$ streptomycin sulfate (Sigma). The culture media were changed twice a week. For the analysis of phase II enzymes induction, the astrocytes collected from $\mathrm{Nrf} 2+/+$ or $\mathrm{Nrf} 2-/-$ mice were treated with sulforaphane (SUL), at $10 \mu \mathrm{mol} / \mathrm{l}$ for $24 \mathrm{~h}$.

\section{Organotypic Spinal Cord Culture}

Organotypic spinal cord culture was prepared as previously described. ${ }^{18}$ Briefly, lumbar spinal cords were collected from 3-day-old mice pups under sterile conditions. The spinal cords were then sectioned transversely at $350-\mathrm{mm}$ intervals using a tissue chopper (Mickle Laboratory Engineering, Surrey, UK). The cultures were maintained at $37^{\circ} \mathrm{C}$ in a humidified incubator with $5 \% \mathrm{CO}_{2}$. One week after collection, the explants were treated with $10 \mu \mathrm{M}$ SUL or vehicle control for $48 \mathrm{~h}$ at $37^{\circ} \mathrm{C}$, and then collected and stored at $-80^{\circ} \mathrm{C}$ for further analysis.

\section{Immunoblotting Analysis}

The lysates were extracted from animal tissues, spinal cord explants, and primary astrocytes by using a total protein extraction kit (Applygen Technologies), according to the manufacturer's instruction. Bradford assay was conducted to determine the protein concentration of each sample. The lysates of each sample $(50 \mu \mathrm{g})$ were run on $10 \%$ SDS-PAGE gel and transferred to PVDF membranes. The membranes were then probed with different primary antibodies, including anti-GCLC and anti-GCLM (Abcam, Cambridge, MA), anti-NQO1, anti-GSS, anti-keap1 and anti-SOD1 (Santa Cruz, CA), and anti-HO-1 (Stressgen Biotechnologies, Victoria, Canada). The bands of interest were detected using an Odyssey Infrared Imaging System (LI-COR, Lincoln, NE). Band intensity was measured using the Odyssey Infrared Imaging System Version 2.1.12 on scanned infrared images. $\beta$-Actin or GAPDH was used as internal control. The original green or red color of a band was converted to black and white colors for data presentation.

\section{Immunofluorescence Staining}

The lumbar spinal cord was post-fixed in $4 \%$ paraformaldehyde for $24 \mathrm{~h}$ and then cryoprotected with 30\% sucrose for $12 \mathrm{~h}$. The tissues were sliced into $30 \mu \mathrm{m}$ sections using a Leica CM1850 freezing microtome. The sections were washed for 3 times in PBS and then perforated with 0.3\% Triton X-100. The tissue sections were double stained by antiGFAP (Chemicon) and anti-NeuN (Santa Cruz) antibodies and detected with corresponding specific fluorophore-conjugated secondary antibodies. The nuclei were counterstained with Hoechst. Slides were mounted and analyzed by fluorescent confocal microscopy (Olympus FV1000).

\section{Immunohistochemistry}

The lumbar spinal cords were post-fixed in $4 \%$ paraformaldehyde, and cut into $20 \mu \mathrm{m}$ sections using Leica VT $1000 \mathrm{~S}$ vibratome. The tissue sections were washed and perforated with $0.3 \%$ Triton X-100. After proper blocking, the tissues were incubated with anti-SMI-32 (Sternberger) and anti-GFAP (Chemicon) antibodies, overnight at $4{ }^{\circ} \mathrm{C}$. The sections were then incubated with Biotin-conjugated secondary antibody (ZSGB-BIO, 1:200) for $2 \mathrm{~h}$ at room temperature (RT), followed by incubation with HRPconjugated streptavidin (ZSGB-BIO, 1:200) for $1 \mathrm{~h}$. The staining was visualized by treating the sections with $0.03 \%$ 
diaminobenzidine for $10 \mathrm{~min}$ at RT. Slides were mounted and analyzed by light microscopy (Olympus BX51).

The numbers of motor neurons (SMI-32 positive) with intact nucleus, $\geq 25 \mu \mathrm{m}$ in diameter and astrocytes (GFAP positive) with intact cell bodies in the anterior horn of lumbar spinal cord were counted.

\section{Glutathione Assay}

Lumbar spinal cords were dissected freshly and snap-frozen in liquid nitrogen. The tissue was then ground in deproteinizing agent and the suspension was centrifuged at $10000 \mathrm{~g}$ for $10 \mathrm{~min}$ at $4{ }^{\circ} \mathrm{C}$. The supernatant was measured for GSH content using a GSH assay kit, according to the manufacturer's instruction (Beyotime Inst Biotech, Jiangsu, China).

\section{Statistical Analysis}

All data are presented as mean \pm s.d. Ages of onset and survival rates were analyzed using Kaplan-Meier analysis. Differences among groups were determined using one-way ANOVA followed by SNK multiple-range test. $P<0.05$ was considered statistically significant. All statistical analysis was conducted using SPSS 16.0 software.

\section{RESULTS}

\section{Significant Upregulation of Cytoprotective Phase II Enzymes in the Lumbar Spinal Cord of Sod1-G93a Mutant Mice}

Oxidative damage is involved in the pathogenesis of ALS. Nrf2 is widely believed to have a critical role in cellular defense against oxidative stress and other toxicities by transcriptionally simulating a wide array of antioxidant proteins and detoxification enzymes via ARE in the target genes. We first examined the expression of several well-known antioxidant genes, including HO-1, NQO1, GCL (both the catalytic subunit GCLC and the regulatory subunit GCLM), and GSS, in lumbar spinal cord and motor cortex of SOD1-G93A transgenic mice at various disease stages. Significant upregulation of HO-1, NQO1, GCLC, and GCLM were detected in the lumbar spinal cord of SOD1-G93A mice at the disease onset and end stages, whereas no significant changes in the expression levels of these proteins were observed in motor cortex of SOD1-G93A mice at either stage (Figure 1a). Quantitative analysis showed 1.5-3.5 fold increases in protein expression of HO-1, NQO1, GCLC, and GCLM were found in the lumbar spinal cord of SOD1-G93A mice, compared with non-transgenic littermates (Figure 1b). Our data suggested that there is severe oxidative stress in the lumbar spinal cord of SOD1-G93A mice, while the motor cortex of those mice is relatively spared.

\section{The Modest Impact of Nrf2 Gene Knockout on Disease Progression in Sod1-G93a Mutant Mice}

Nrf2 activators were shown to be neuroprotective and able to slow disease progression in a mouse model of ALS. ${ }^{12}$ To further study the involvement of Nrf2 pathway in the process
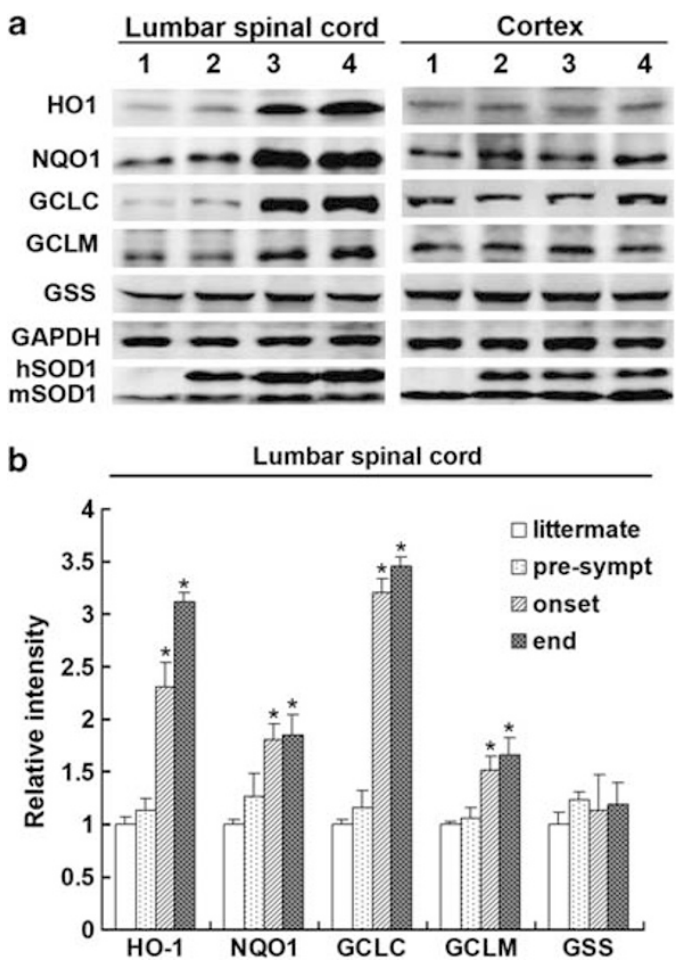

Figure 1 Induction of phase II enzymes in the lumbar spinal cord of SOD1-G93A mice. (a) Immunoblotting images showing the expressions of phase II enzymes (heme oxygenase-1 (HO-1), NAD(P)H: quinone oxidoreductase 1 (NQO1), catalytic subunit of glutamate cysteine ligase (GCLC), GCLM, and GSS) in the lumbar spinal cord and motor cortex of SOD1-G93A mice at different disease stages. 1, littermate; 2 , presymptomatic stage (60 days of age); 3 , onset stage; and 4 , end stage. (b) Quantitative densitometry showing the expression of phase II enzymes in the lumbar spinal cord. Three females and three males were examined at each stage. Data were obtained from three independent experiments. ${ }^{*} P<0.05$, compared with littermate.

of motor neuron degeneration in SOD1-G93A mice, we generated double mutant mice, Nrf2 - / - SOD1-G93A, by mating SOD1-G93A mice to Nrf2-/- animals with the same genetic background. The Nrf2 gene knockout was confirmed by PCR examination (Figure 2a). As shown in Figures $2 \mathrm{~b}$ and $\mathrm{c}$, SOD1-G93A/Nrf2 - / - mice exhibited earlier disease onset and shorter life span, compared with SOD1-G93A/Nrf2 $+/+$ mice. The disease onset age of SOD1-G93A/Nrf2 - / - mice was $88.0 \pm 7.7$ days (male) and $95.4 \pm 4.9$ days (female), compared with $98.5 \pm 3.2$ days and $102.9 \pm 5.1$ days for male and female SOD1-G93A/Nrf2 + / + mice, respectively. The average life span of SOD1-G93A/ Nrf2 - / - mice was $108.5 \pm 8.5$ days for male and $115.9 \pm 9.9$ days for female, compared with $114.0 \pm 7.6$ days and $123.0 \pm 9.8$ days for male and female SOD1-G93A/ $\mathrm{Nrf} 2+/+$ mice, respectively (Table 1 ). Despite of the earlier disease onset and shorter life span observed in SOD1-G93A/ Nrf2 - / - mice, we found similar motor neuron loss and astrocytosis in the anterior horn of lumbar spinal cord at the end stage between SOD1-G93A/Nrf2 $+/+$ mice and SOD1G93A/Nrf2 - / - mice (Figure 2d). 


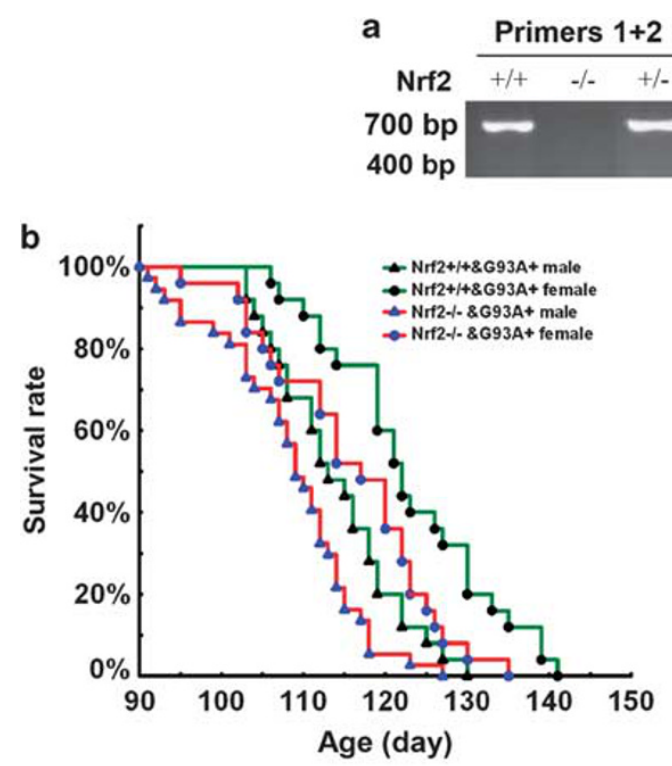

\begin{tabular}{lr} 
Primers & $1+3$ \\
\hline$+/+\quad-/-\quad+/-$
\end{tabular}
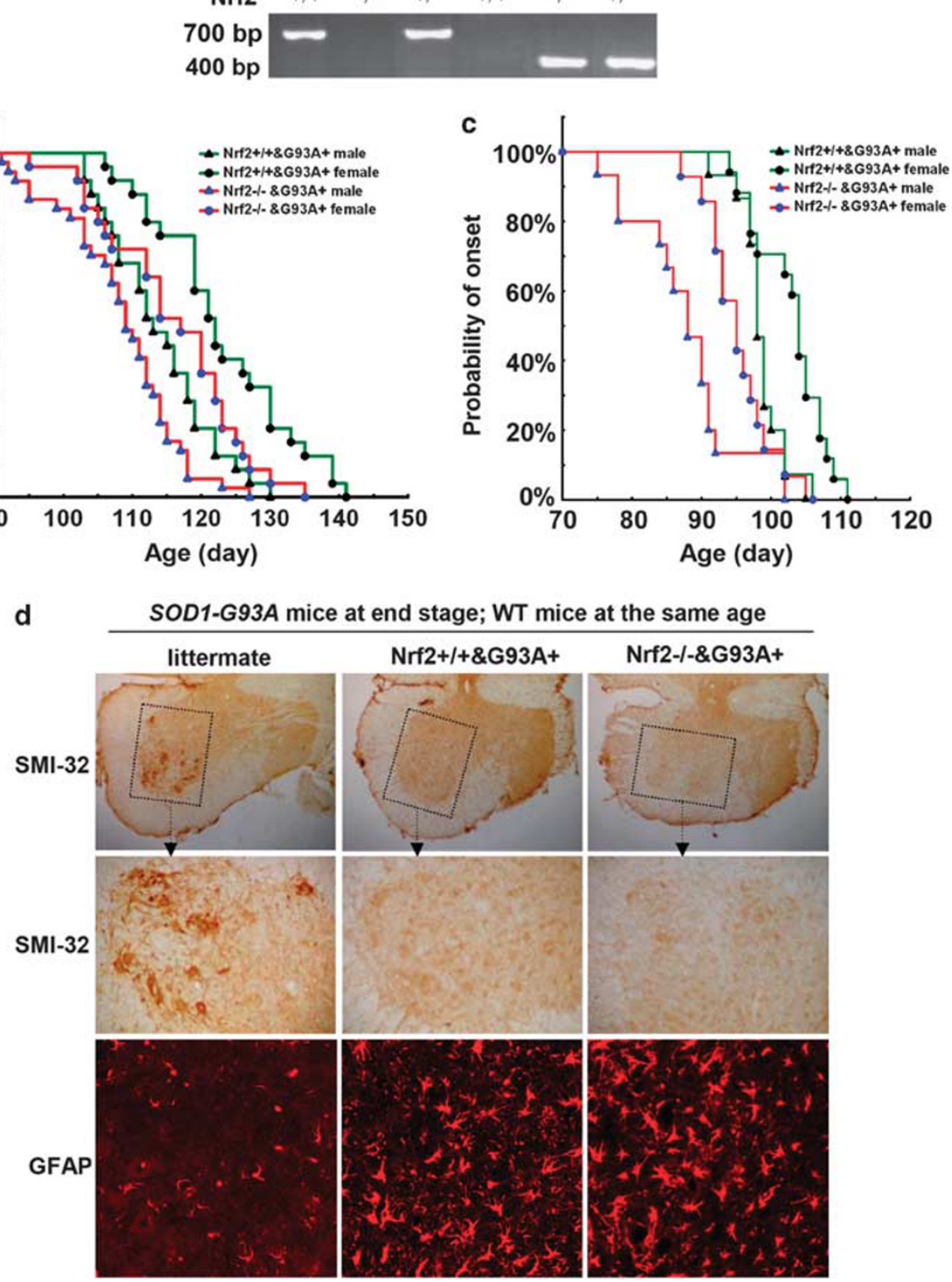

Figure 2 Effect of nuclear factor erythroid 2-related factor 2 (Nrf2) knockout on disease progression of SOD1-G93A mice. (a) PCR identification of Nrf2 knockout mice. (b) Survival rate of Nrf2 + / SOD1-G93A and Nrf2 - / - SOD1-G93A mice. (c) Probability of disease onset of Nrf2 + / SOD1-G93A and Nrf2 - / - SOD1-G93A mice. (d) Immunohistochemical analysis of motor neuron loss and immunofluorescence analysis of astrocytic proliferation in the lumbar spinal cord of Nrf2 + / SOD1-G93A and Nrf2 - / - SOD1-G93A mice. SMI-32 and GFAP were used as markers for motor neuron and astrocytes, respectively. Data were representative of three females and three males in each group.

\section{The Effect of Nrf2 Knockout on Motor Neuron Survival and Astrocytic Proliferation}

ALS is pathologically characterized by motor neuron loss, and activated astrocytes have an vital role in the induction and propagation of motor neuron loss. ${ }^{19}$ To examine the pathological changes in the lumbar spinal cord of SOD1G93A mice after Nrf2 knockout, SMI-32 and GFAP immunohistochemistry were performed on the lumbar spinal cord tissues collected from SOD1-G93A/Nrf2 - / and SOD1-G93A/Nrf2 $+/+$ mice at various stages (pre- symptomatic and onset stage). Significant motor neuron loss was observed in SOD1-G93A/Nrf2 $-/-$ mice at presymptomatic stage, whereas neuronal loss with similar extent was not detected till onset stage in SOD1-G93A/ $\mathrm{Nrf2}+/+$ mice. Astrocytic proliferation was significant at 60 days of age and became more prominent at $88-95$ days of age in both SOD1-G93A/Nrf2 + / + and SOD1-G93A/Nrf2 - / mice. However, there were more proliferating astrocytes found in SOD1-G93A/Nrf2 - / - mice compared with SOD1-G93A/Nrf2 + I + animals (Figure 3). 
Table 1 Life span and age of onset of Nrf2 + I+ \&G93A/F2 and Nrf2 - I - \&G93A/F2 mice

\begin{tabular}{|c|c|c|c|c|}
\hline \multirow[b]{2}{*}{ Group } & \multicolumn{2}{|c|}{ Life span } & \multicolumn{2}{|c|}{ Age of onset } \\
\hline & Male & Female & Male & Female \\
\hline $\mathrm{Nrf2}+/+\& \mathrm{G} 93 \mathrm{~A}+/ \mathrm{F2}$ (number of mice) & $114.0 \pm 7.6(25)$ & $123.0 \pm 9.8(25)$ & $98.5 \pm 3.2(15)$ & $102.9 \pm 5.1(17)$ \\
\hline $\mathrm{Nrf2}-/-\& \mathrm{G} 93 \mathrm{~A}+/ \mathrm{F2}$ (number of mice) & $108.5 \pm 8.5^{*}(37)$ & $115.9 \pm 9.9^{*}(25)$ & $88.0 \pm 7.7^{*}(15)$ & $95.4 \pm 4.9 *(14)$ \\
\hline
\end{tabular}

Wild-type $(\mathrm{Nrf2}+/+)$ and Nrf2-deficient (Nrf2-/-) CD1/ICR female mice were crossed to B6SJL-Tg (SOD1-G93A) 1Gur/J male mice. SOD1-G93A transgenic male mice in the F1 generation were backcrossed to Nrf2 +/+ and Nrf2 - / - CD1/ICR female mice. In the F2 generation, Nrf2 + / + and SOD1-G93A transgenic mice (SOD1-G93A/Nrf2 +/ +/F2), and Nrf2 - / - and SOD1-G93A transgenic mice (SOD1-G93A/Nrf2 - / - /F2) were generated. 43 Nrf2 + / + \&G93A/F2 mice from 14 litters and 58 SOD1-G93A/Nrf2 - / - /F2 mice from 31 litters were analyzed for life span. Thirty-two SOD1-G93A/Nrf2 + / + /F2 mice from 12 litters and 29 SOD1-G93A/Nrf2 - / - /F2 mice from 20 litters were analyzed for age of disease onset. ${ }^{*} P<0.05$, compared with SOD1-G93A/Nrf2 $+/+/$ F2 mice of the same sex.

\section{The Effect of Nrf2 Knockout on the Induction of Phase II Enzymes in Sod1-G93a Mice}

Activation of Nrf2-ARE pathway has been shown to be protective against oxidative stress-induced cell death. ${ }^{20}$ Several lines of evidences have suggested that the induction of Nrf2-regulated phase II enzymes, including HO-1, NQO1, and GCLM, etc, had strongly protective effects against oxidative damage and neuronal death. ${ }^{18,21}$ In addition, it was reported that GCLM knockout decreased the life span of SOD1-G93A mice by $55 \%$, which was related to the reduction of GSH. ${ }^{22}$ To better understand the role of Nrf2 in ALS, we examined the expression of several phase II enzymes in different tissues, including lumbar spinal cord and motor cortex, of Nrf2 $+/+$ and Nrf2 - / - SOD1-G93A mice (Figure 4). Among the five enzymes examined (HO-1, NQO1, GCLC, GCLM, and GSS), the expressions of four enzymes were significantly increased (1.8-4.3 fold) in the lumbar spinal cord of Nrf2 $+/+$ SOD1-G93A mice compared with that of non-transgenic Nrf2 $+/+$ littermates, except for GSS. Interestingly, three of the examined enzymes, including HO-1, GCLC, and GCLM, were significantly induced (2.3-4.2 fold) in Nrf2 - / - SOD1-G93A mice as well, compared with the Nrf2-/- littermates. Further analysis showed that the induction of NQO1 in lumbar spinal cord of SOD1-G93A/Nrf2 $+1+$ mice was significantly higher than that of SOD1-G93A/Nrf2 - / - mice. However, the expressions of four other examined phase II enzymes in lumbar spinal cord remain unchanged after Nrf2 knockout in SOD1-G93A mice (Figure 4). No obvious motor neuron loss and expression changes of HO-1, NQO1, GCLC, GCLM, and GSS were observed in the motor cortex of Nrf2 - / - SOD1G93A mice compared with Nrf2 + / SOD1-G93A mice (Supplementary Figure 1).

As Keap1 sequesters Nrf2 in the cytoplasm and is an important modulator of Nrf2/ARE pathway, we further examined the expression of keap1. We found significant upregulation of keap1 in both $\mathrm{Nrf} 2+/+$ and $\mathrm{Nrf} 2-/-$ SOD1-G93A mice at end stage compared with non-transgenic littermates, while the former showed much higher keap1 induction than the latter (Figures $4 \mathrm{c}$ and $\mathrm{d}$ ).
GSH has a key role in cellular redox signaling and protecting against oxidative injury, ${ }^{23}$ thereby, we examined the GSH levels in different tissues of $\mathrm{Nrf} 2+1+$ and $\mathrm{Nrf2}-/-$ mice. Interestingly, there was significantly less GSH in the liver, bladder, and lung of $\mathrm{Nrf} 2-1-$ mice, compared with $\mathrm{Nrf} 2+/+$ mice. However, GSH content in lumbar spinal cord, motor cortex, and kidney was comparable between $\mathrm{Nrf} 2-I-$ and $\mathrm{Nrf} 2+I+$ mice (Figures $5 \mathrm{a}$ and $\mathrm{b}$ ).

\section{Role of Nrf2 in Phase II Enzymes Induction in Cultured Primary Astrocytes and Spinal Cord Explants}

The role of Nrf2 in phase II enzymes induction was further studied in in-vitro models. Astrocytes are the major reactive glial cells companying motor neuron loss in SOD1-G93A transgenic mice. ${ }^{24}$ In the current study, we also examined the effect of Nrf2 on the induction of phase II enzymes in astrocytes. Primary astrocytes isolated from the brain cortex of $\mathrm{Nrf} 2+/+$ and $\mathrm{Nrf} 2-/-$ mice were treated with Nrf2ARE activator, SUL. SUL induced robust upregulation of HO-1, NQO1, and GCLM in Nrf2 + / + astrocytes, and it also significantly upregulate the expression of $\mathrm{HO}-1$ in Nrf2 - / - cells. However, the SUL-induced upregulations of NQO1 and GCLM were abolished in Nrf2 - / - astrocytes. The GCLC and GSS expressions were not altered in either type of astrocytes by the treatment of SUL, although Nrf2 - / - cells expressed somewhat lower basal level of GSS than Nrf2 $+/+$ cells (Figures $6 \mathrm{a}$ and $\mathrm{b}$ ). These data suggested that Nrf2 pathway is associated with NQO1 and GCLM induction in astrocyte, but it might not regulate the expression of HO-1, GCLC, and GSS in those cells. The Nrf2-independent HO-1 and Nrf2-dependent NQO1 induction in astrocytes by SUL are in compliance with our in-vivo findings (Figure 4). Similar studies were also conducted on spinal cord explants. We found that SUL induced significant upregulation of NQO1 in an Nrf2-dependent manner, whereas the expression of GCLC was not altered by the SUL treatment and no difference was observed between $\mathrm{Nrf} 2+/+$ mice and Nrf2 $-/-$ mice (Figure $6 \mathrm{c}$ ). 
a
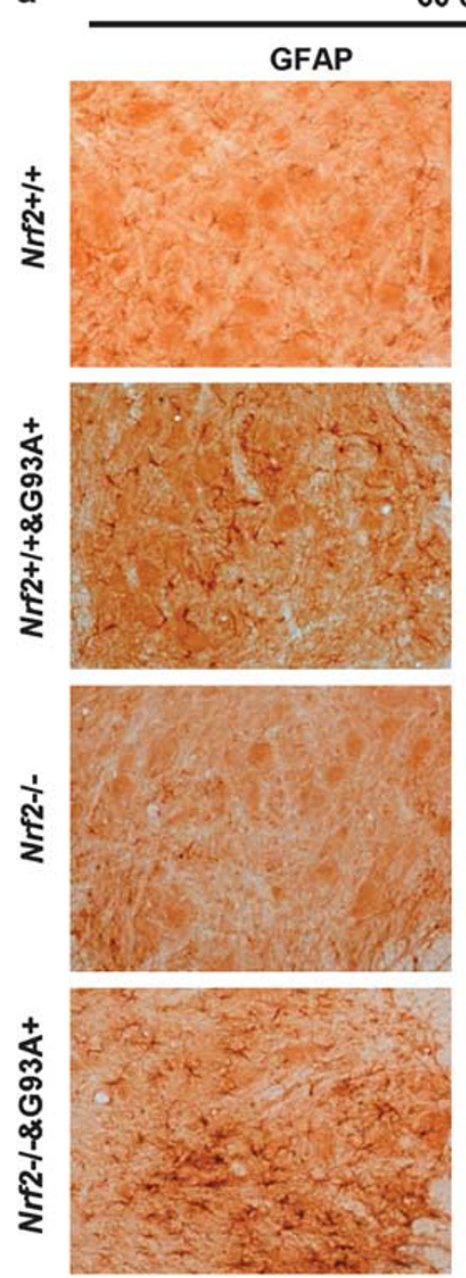

60 days
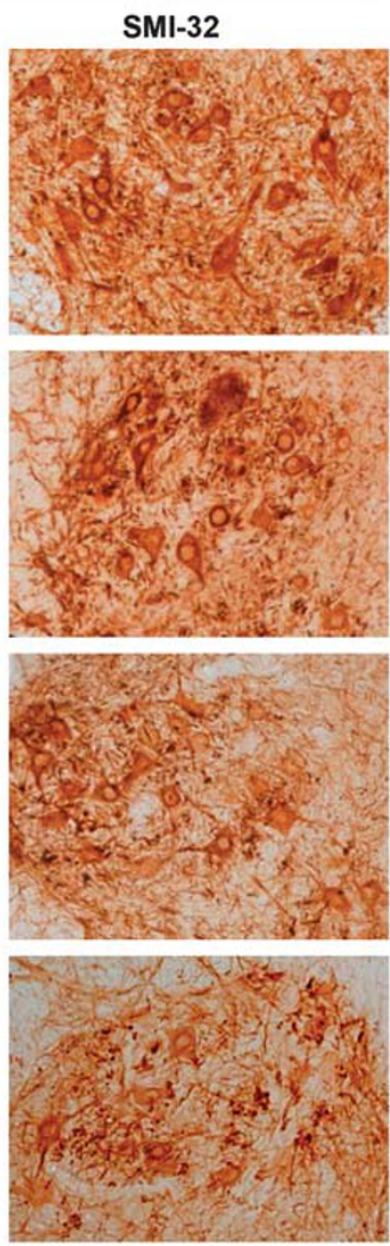
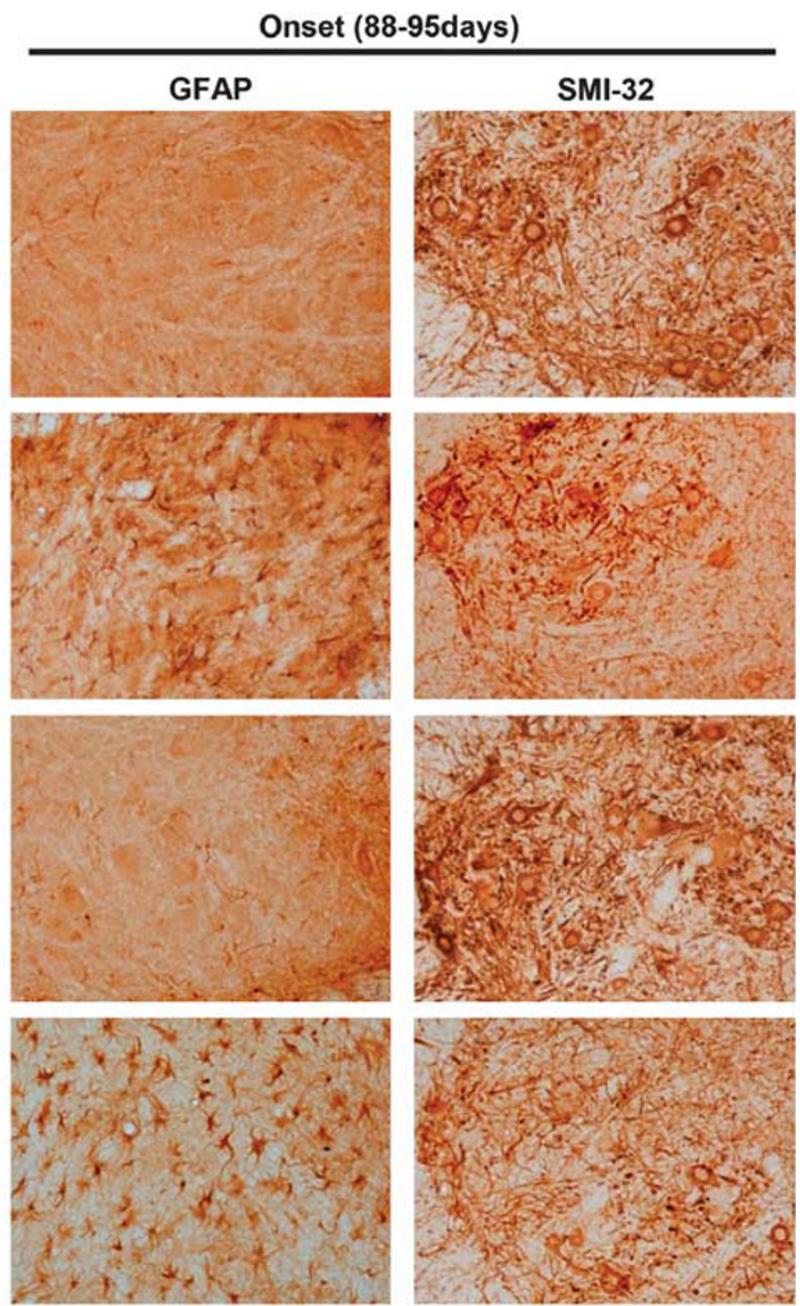

b
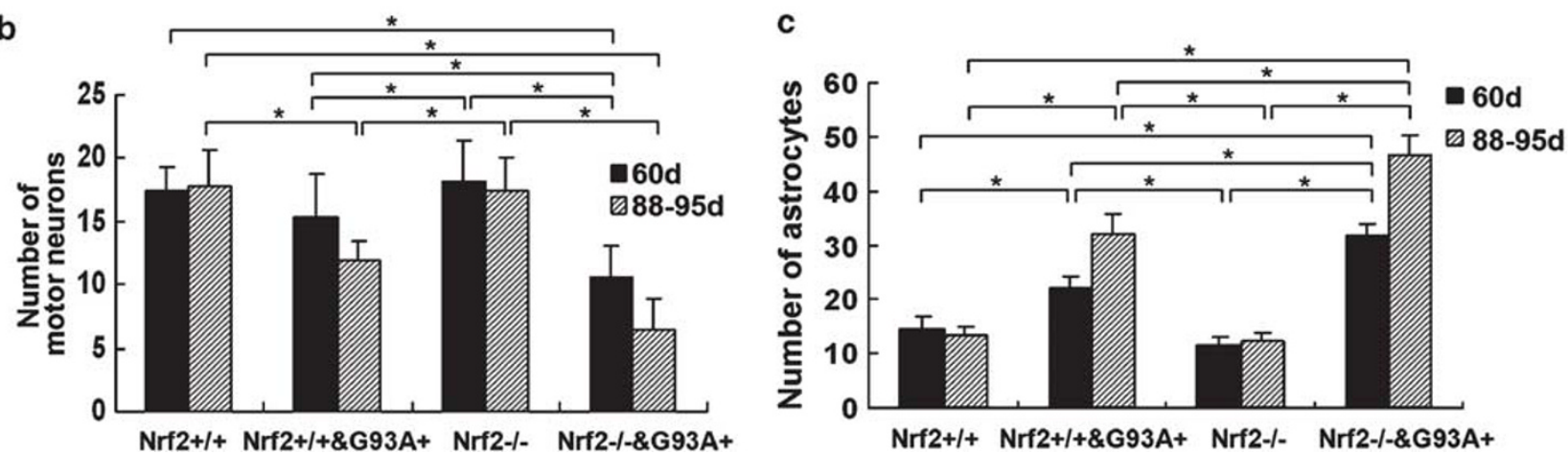

Figure 3 Earlier motor neuron loss and astrocytic proliferation in nuclear factor erythroid 2-related factor 2 (Nrf2) - / - SOD1-G93A mice. (a) Micrographs showing motor neurons (SMI-32) and astrocytes (GFAP) in the lumbar spinal cord of Nrf2 + / + SOD1-G93A, Nrf2 - / - SOD1-G93A mice and their littermates at 60 and 88-95 days of age. (b) Quantitative analysis of motor neuron numbers. (c) Quantitative analysis of astrocyte numbers. ${ }^{*} P<0.05$. Data are representative of three males in each group.

\section{DISCUSSION}

Nrf2-mediated regulation of cellular antioxidant production has an important role in neuroprotection against some neurodegenerative diseases. Activation of Nrf2 pathway promotes the transcription of various cytoprotective genes, including HO-1, NQO1, and GCLC, etc. HO-1 is highly inducible by various pro-oxidant and other stressors. ${ }^{25}$ Upregulated HO-1 expression has been found in the brain of patients with $\mathrm{AD},{ }^{26}$ and in the lumbar spinal cord of SOD1-G93A transgenic mice. ${ }^{15}$ NQO1, a redox-regulated flavoenzyme, is sensitive in monitoring cellular redox state. ${ }^{27}$ GCLC, GCLM, and GSS are enzymes essential for synthesis of 


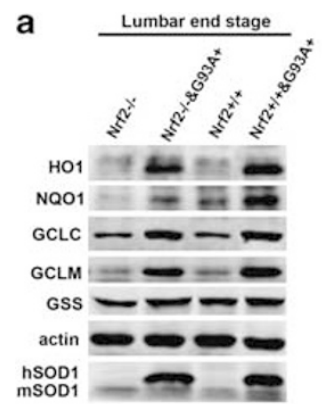

b

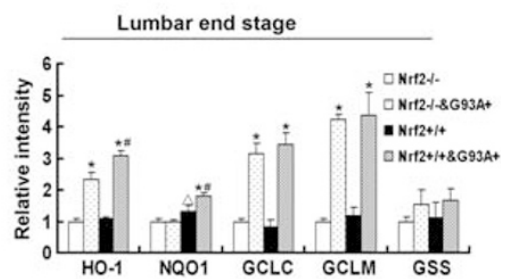

C
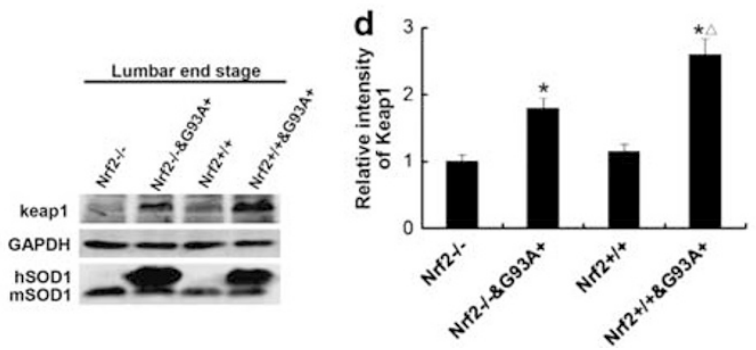

Figure 4 Phase II enzyme inductions and keap1 expression in the spinal cord of nuclear factor erythroid 2-related factor 2 (Nrf2) + / + SOD1-G93A and Nrf2 - / - SOD1-G93A mice. (a) Immunoblot analysis of phase II enzymes. Inductions of heme oxygenase-1 ( $\mathrm{HO}-1), \mathrm{NAD}(\mathrm{P}) \mathrm{H}$ : quinone oxidoreductase 1 (NQO1), catalytic subunit of glutamate cysteine ligase (GCLC), GCLM, and GSS in the lumbar spinal cord were compared between Nrf2 + / + SOD1-G93A and Nrf2 - / - SOD1-G93A mice at end stage. Data were representative of three females and three males in each group. (b) Histograms of phase II enzyme expressions in a. ${ }^{*} P<0.05$, compared with non-transgenic littermate; $\triangle P<0.05$, compared with Nrf2 - / - mice; and ${ }^{\#} P<0.05$, compared with Nrf2 - / - SOD1-G93A mice. (c) Changes of keap1 expression in the lumbar spinal cord of Nrf2 + / + SOD1-G93A and Nrf2 - / - SOD1-G93A mice at end stage. Data were representative of three independent experiments. (d) Histograms of keap1 expression in c. ${ }^{*} P<0.05$, compared with non-transgenic littermate; $\triangle P<0.05$, compared with Nrf2 - / SOD1-G93A mice.

GSH, which is the major endogenous thiol antioxidant, counteracting oxidative injury within cells. ${ }^{23}$ Upregulation of HO-1, NQO1, GCLC, and GCLM in the lumbar spinal cord of SOD1-G93A mice implicates that the affected tissues are experiencing chronic oxidative stress. Although the expression of Nrf2-mediated antioxidant genes confers cytoprotection, longer-term induction of some enzymes such as HO-1 may exacerbate oxidative injury to the mitochondria and the microtubular network. ${ }^{28}$

Nrf2 and its downstream ARE-driven genes have a very important role in protecting cells from the insults of oxidative stress. ${ }^{29}$ Several studies demonstrated that Nrf2 knockout deteriorate the oxidative damage under certain circumstance. $^{30,31}$ However, in the current study, only modest impacts on disease progression were found with the absence of Nrf2 activity in SOD1-G93A mice. The induction of ARE-driven genes is required for the Nrf2-mediated neuroprotection, and the knockout of some ARE-driven genes, such as CCS and GCLM, had dramatic effects (significantly decreased survival) in ALS animal model. ${ }^{22,32}$
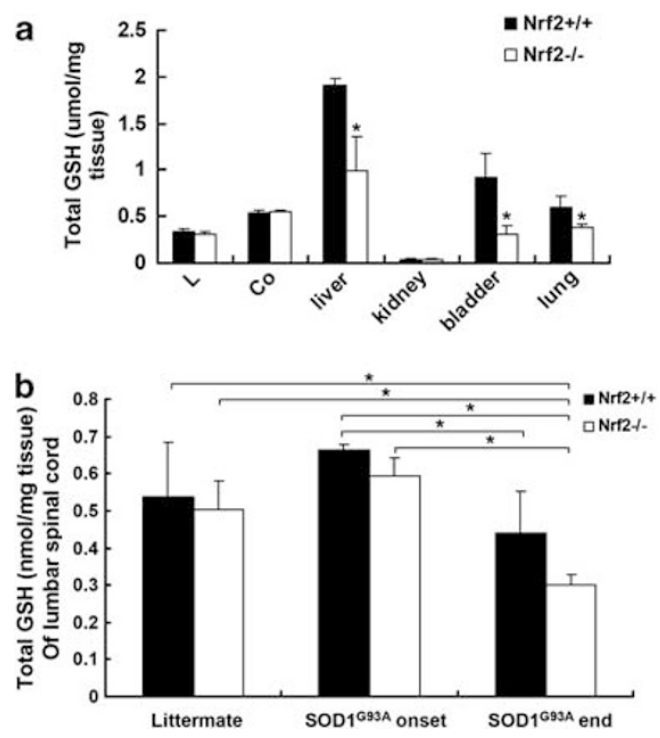

Figure 5 Changes of glutathione (GSH) content in the spinal cord of nuclear factor erythroid 2-related factor 2 (Nrf2) + / SOD1-G93A and Nrf2 - / - SOD1-G93A mice. (a) Total GSH content in different tissues of $\mathrm{Nrf} 2+/+$ and $\mathrm{Nrf} 2-/$ - mice. ${ }^{*} P<0.05$, compared with the same tissue of $\mathrm{Nrf2}+/+$ mice. Data are representative of three females at 90 days of age. (b) Total GSH content in the lumbar spinal cord of non-transgenic littermates (two females and two males), Nrf2 + / + SOD1-G93A, Nrf2 - / - SOD1-G93A mice at onset (one female and two males) and end stages (two females and one male). ${ }^{*} P<0.05$.

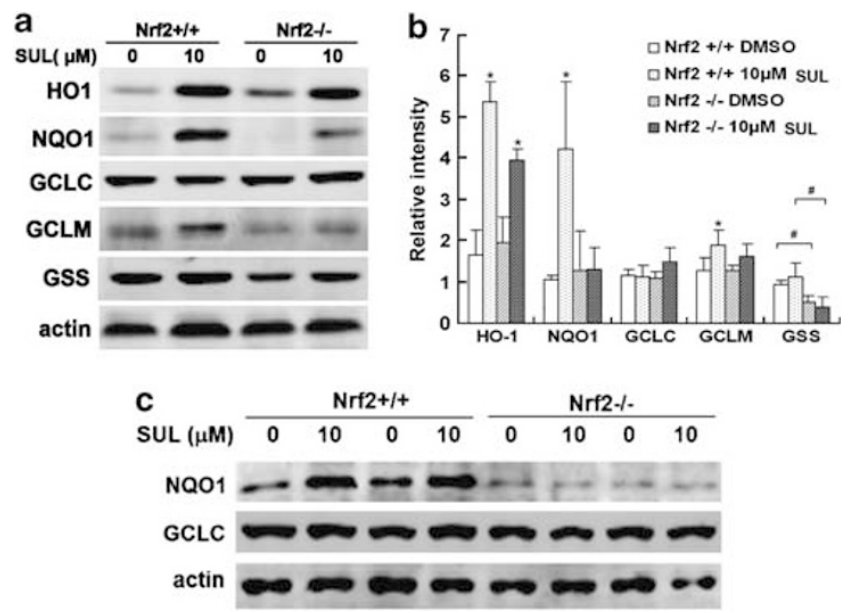

Figure 6 Induction of phase II enzymes in cultured astrocytes and spinal cord explants treated with sulforaphane (SUL). (a) Immunoblot analysis of phase II enzyme inductions in nuclear factor erythroid 2-related factor $2(\mathrm{Nrf2})+/+$ and Nrf2 - / - astrocytes treated with $10 \mu \mathrm{M}$ SUL for $24 \mathrm{~h}$. Data are representative of three independent experiments. (b) Histograms of phase II enzyme expressions in a. ${ }^{*} P<0.05$, compared with untreated group. ${ }^{\#} P<0.05$, compared with Nrf2 $+/+$ group with or without $10 \mu \mathrm{M}$ SUL treatment. (c) NAD(P)H: quinone oxidoreductase 1 (NQO1) was induced by SUL in the spinal cord explants from Nrf2 $+/+$ and Nrf2 + / SOD1-G93A mice, but not Nrf2 - / - and Nrf2 - / SOD1-G93A mice. Total extracts of lumbar spinal cord explants were examined for the expression levels of NQO1 and catalytic subunit of glutamate cysteine ligase (GCLC) by western blotting. $\beta$-Actin was used as a control. The results are representative of triplicate experiments. HO-1, heme oxygenase-1. 
We found that NQO1 induction was disabled by Nrf2 knockout, whereas, the induction of HO-1, GCLC, and GCLM does not differ between Nrf2 - / - SOD1-G93A and $\mathrm{Nrf} 2+/+$ SOD1-G93A mice. Consequently, the GSH content in lumbar spinal cord was not altered in Nrf2 - / mice compared with controls. The modest impacts on GSH level and the induction of ARE-driven genes by $\mathrm{Nrf2}$ knockout explain the mild change in the course of disease found in Nrf2 - / - SOD1-G93A mice. SUL is a well-known chemopreventive phytochemical, capable of activating Nrf2regulated cytoprotective signaling pathway. ${ }^{33}$ Our results showed that SUL induced upregulation of NQO1 in an Nrf2-dependent manner, however, Nrf2 activation by SUL does not lead to induction of GCLC and GSS, even though GCLC gene promoter is known to carry a functional ARE. Similar modulation of these phase II enzymes was observed during keratinocyte differentiation as well. ${ }^{34}$ These results suggested that other mechanisms besides Nrf2 pathway might be involved in the induction of the antioxidant genes in this ALS mouse model. Indeed, previous study showing the induction of HO-1 in Nrf2-deficient MEF and HEK-293 cells provided evidence that $\mathrm{HO}-1$ can be regulated by Nrf2independent mechanisms. ${ }^{35}$ Moreover, it has been reported that Nrf1, another member of CNC family transcription factors, may compensate for the deficiency of Nrf2. ${ }^{36,37}$

The breeding strategy we used by inbreeding SOD1-G93A/ $\mathrm{Nrf} 2+/+$ and SOD1-G93A/Nrf2 - / - mice separately without mixing may contribute to the moderate difference in disease progression. Furthermore, the main non-neuronal cells in the central nervous system are astrocytes, which contribute to the maintenance of normal neuronal function. Astrocytes isolated from SOD1-G93A mice are toxic to cocultured motor neurons. ${ }^{38}$ On the other hand, a recent study suggested that $\mathrm{Nrf2}$ overexpression in astrocytes significantly delayed onset and extended survival of SOD1G93A mice. ${ }^{39}$ GST secretion by astrocytes has been demonstrated to be protective for neurons against oxidative stress. ${ }^{40}$ The Nrf2 gene was genetically depleted from both neurons and astrocytes in the double mutant animals generated in our current study, and the depletion of Nrf2 in the proliferated astrocytes can exacerbate the toxicity to motor neurons and contribute to the deteriorative motor neuron loss in Nrf2 - / - SOD1-G93A mice.

Similar phenomena were observed in cultured spinal cord slices. Anyhow, different cell types under different settings may contribute to the in-vivo and in-vitro differences in phase II enzyme induction.

We demonstrated here that there was marked induction of phase II enzymes in the lumbar spinal cord of SOD1-G93A mice compared with non-transgenic littermates, while no induction was observed in motor cortex. Nrf2 knockout led to accelerated motor neuron loss and astrocytic proliferation, and caused earlier disease onset and shorter life span in SOD1-G93A transgenic mice. However, the effect of Nrf2 knockout is relatively modest. Our data show that the induction of antioxidative genes, except $\mathrm{NQO}$, such as $\mathrm{HO}-1$ and GCL, and the tissue GSH level are independent of Nrf2 activity, which may contribute at least in part to the limited effect of Nrf2 knockout on this ALS animal model. Taken together, our findings suggest that Nrf2 is not the key mediator regulating protections against SOD1 mutant-induced chronic neurodegeneration in this ALS animal model, and further investigations are granted to study the precise neuroprotective mechanisms in ALS and determine the potential targets for designing more effective therapeutic approach.

Supplementary Information accompanies the paper on the Laboratory Investigation website (http://www.laboratoryinvestigation.org)

\section{ACKNOWLEDGEMENTS}

We thank Yongbo Sui for his technical supports and Dr Thomas W. Kensler (John Hopkins University, Baltimore, MD, USA) for providing us the breeders of Nrf2-deficient mice. This work was supported by the Natural Science Foundation of China (grant numbers 30900460 and 81171210) and the Science and Technological Department of Hebei Province (grant number 11966122D)

\section{DISCLOSURE/CONFLICT OF INTEREST}

The authors declare no conflict of interest.

1. Rowland LP. Amyotrophic lateral sclerosis. Curr Opin Neurol 1994;7:310-315.

2. Rosen $\mathrm{DR}$, Siddique $\mathrm{T}$, Patterson $\mathrm{D}$, et al. Mutations in $\mathrm{Cu} / \mathrm{Zn}$ superoxide dismutase gene are associated with familial amyotrophic lateral sclerosis. Nature 1993;362:59-62.

3. Gurney ME, Pu H, Chiu AY, et al. Motor neuron degeneration in mice that express a human $\mathrm{Cu}, \mathrm{Zn}$ superoxide dismutase mutation. Science 1994;264:1772-1775.

4. Ferrante RJ, Browne SE, Shinobu LA, et al. Evidence of increased oxidative damage in both sporadic and familial amyotrophic lateral sclerosis. J Neurochem 1997e;69:2064-2074.

5. Drechsel DA, Estevez AG, Barbeito $L$, et al. Nitric oxide-mediated oxidative damage and the progressive demise of motor neurons in ALS. Neurotox Res 2012;22:251-264.

6. Barber SC, Shaw PJ. Oxidative stress in ALS: key role in motor neuron injury and therapeutic target. Free Radic Biol Med 2010;48:629-641.

7. Itoh K, Chiba T, Takahashi S, et al. An Nrf2/small Maf heterodimer mediates the induction of phase II detoxifying enzyme genes through antioxidant response elements. Biochem Biophys Res Commun 1997e;236:313-322.

8. Gong $\mathrm{P}$, Stewart $\mathrm{D}, \mathrm{Hu}$ B, et al. Activation of the mouse heme oxygenase-1 gene by 15 -deoxy-Delta(12,14)- prostaglandin $J(2)$ is mediated by the stress response elements and transcription factor Nrf2. Antioxid Redox Signal 2002e;4:249-257.

9. Kim HJ, Zheng M, Kim SK, et al. CO/HO-1 induces NQO-1 expression via Nrf2 activation. Immune Netw 2011;11:376-382.

10. Chan K, Han XD, Kan YW. An important function of Nrf2 in combating oxidative stress: detoxification of acetaminophen. Proc Natl Acad Sci USA 2001e;98:4611-4616.

11. Alam J, Stewart D, Touchard C, et al. Nrf2, a Cap'n'Collar transcription factor, regulates induction of the heme oxygenase-1 gene. J Biol Chem 1999e;274:26071-26078.

12. Neymotin A, Calingasan NY, Wille $E$, et al. Neuroprotective effect of Nrf2/ARE activators, CDDO ethylamide and CDDO trifluoroethylamide, in a mouse model of amyotrophic lateral sclerosis. Free Radic Biol Med 2011:51:88-96.

13. Guo Y, Zhang K, Wang Q, et al. Neuroprotective effects of diallyl trisulfide in SOD1-G93A transgenic mouse model of amyotrophic lateral sclerosis. Brain Res 2011;1374:110-115.

14. Mimoto T, Miyazaki K, Morimoto $\mathrm{N}$, et al. Impaired antioxydative Keap1/Nrf2 system and the downstream stress protein responses in the motor neuron of ALS model mice. Brain Res 2012;1446:109-118. 
15. Guo Y, Duan W, Li Z, et al. Decreased GLT-1 and increased SOD1 and HO-1 expression in astrocytes contribute to lumbar spinal cord vulnerability of SOD1-G93A transgenic mice. FEBS Lett 2010;584: $1615-1622$.

16. Weydt $\mathrm{P}$, Hong SY, Kliot $M$, et al. Assessing disease onset and progression in the SOD1 mouse model of ALS. Neuroreport 2003e; 14:1051-1054.

17. Duan W, Zhang R, Guo Y, et al. Nrf2 activity is lost in the spinal cord and its astrocytes of aged mice. In Vitro Cell Dev Biol Anim 2009;45:388-397.

18. $\mathrm{Li} \mathrm{CY}$, Liu XY, Bu H, et al. Prevention of glutamate excitotoxicity in motor neurons by 5,6-dihydrocyclopenta-1,2-dithiole-3-thione: implication to the development of neuroprotective drugs. Cell Mo Life Sci 2007e;64:1861-1869.

19. Barbeito LH, Pehar $M$, Cassina $P$, et al. A role for astrocytes in motor neuron loss in amyotrophic lateral sclerosis. Brain Res Brain Res Rev 2004e;47:263-274.

20. Johnson JA, Johnson DA, Kraft AD, et al. The Nrf2-ARE pathway: an indicator and modulator of oxidative stress in neurodegeneration. Ann N Y Acad Sci 2008e;1147:61-69.

21. Scapagnini G, Vasto S, Abraham NG, et al. Modulation of Nrf2/ARE pathway by food polyphenols: a nutritional neuroprotective strategy for cognitive and neurodegenerative disorders. Mol Neurobiol 2011;44192-201.

22. Vargas MR, Johnson DA, Johnson JA. Decreased glutathione accelerates neurological deficit and mitochondrial pathology in familial ALS-linked hSOD1(G93A) mice model. Neurobiol Dis 2011;43:543-551.

23. Dickinson DA, Forman HJ. Glutathione in defense and signaling: lessons from a small thiol. Ann N Y Acad Sci 2002e:973:488-504.

24. Levine JB, Kong J, Nadler M, et al. Astrocytes interact intimately with degenerating motor neurons in mouse amyotrophic lateral sclerosis (ALS). Glia 1999;28:215-224.

25. Schipper HM, Song $W$, Zukor $H$, et al. Heme oxygenase-1 and neurodegeneration: expanding frontiers of engagement. J Neurochem 2009e;110:469-485.

26. Schipper HM, Cisse S, Stopa EG. Expression of heme oxygenase-1 in the senescent and Alzheimer-diseased brain. Ann Neurol 1995e;37:758-768.

27. Raina AK, Templeton DJ, Deak JC, et al. Quinone reductase (NQO1), a sensitive redox indicator, is increased in Alzheimer's disease. Redox Rep 1999e;4:23-27.

28. Stahnke T, Stadelmann C, Netzler A, et al. Differential upregulation of heme oxygenase-1 (HSP32) in glial cells after oxidative stress and in demyelinating disorders. J Mol Neurosci 2007e;32:25-37.
29. Yang $\mathrm{C}$, Zhang $\mathrm{X}$, Fan $\mathrm{H}$, et al. Curcumin upregulates transcription factor Nrf2, HO-1 expression and protects rat brains against focal ischemia. Brain Res 2009e;1282:133-141.

30. Wruck CJ, Fragoulis A, Gurzynski A, et al. Role of oxidative stress in rheumatoid arthritis: insights from the Nrf2-knockout mice. Ann Rheum Dis 2011;70:844-850.

31. Hong Y, Yan W, Chen S, et al. The role of Nrf2 signaling in the regulation of antioxidants and detoxifying enzymes after traumatic brain injury in rats and mice. Acta Pharmacol Sin 2010;31:1421-1430.

32. Son $M$, Puttaparthi $K$, Kawamata $H$, et al. Overexpression of CCS in G93A-SOD1 mice leads to accelerated neurological deficits with severe mitochondrial pathology. Proc Natl Acad Sci USA 2007e; 104:6072-6077.

33. Zhang Y, Tang L. Discovery and development of sulforaphane as a cancer chemopreventive phytochemical. Acta Pharmacol Sin 2007e;28:1343-1354.

34. Piao MS, Park JJ, Choi JY, et al. Nrf2-dependent and Nrf2independent induction of phase 2 detoxifying and antioxidant enzymes during keratinocyte differentiation. Arch Dermatol Res 2012;304:387-395.

35. Wright MM, Kim J, Hock TD, et al. Human haem oxygenase-1 induction by nitro-linoleic acid is mediated by CAMP, AP-1 and E-box response element interactions. Biochem J 2009e;422:353-361.

36. Zhao R, Hou $Y$, Xue $P$, et al. Long isoforms of NRF1 contribute to arsenic-induced antioxidant response in human keratinocytes. Environ Health Perspect 2011:119:56-62.

37. Braun S, Hanselmann C, Gassmann MG, et al. Nrf2 transcription factor, a novel target of keratinocyte growth factor action which regulates gene expression and inflammation in the healing skin wound. Mol Cell Biol 2002e;22:5492-5505.

38. Nagai $M$, Re DB, Nagata $T$, et al. Astrocytes expressing ALS-linked mutated SOD1 release factors selectively toxic to motor neurons. Nat Neurosci 2007e;10:615-622.

39. Vargas MR, Johnson DA, Sirkis DW, et al. Nrf2 activation in astrocytes protects against neurodegeneration in mouse models of familial amyotrophic lateral sclerosis. J Neurosci 2008e;28: 13574-13581.

40. Kraft AD, Johnson DA, Johnson JA. Nuclear factor E2-related factor 2dependent antioxidant response element activation by tertbutylhydroquinone and sulforaphane occurring preferentially in astrocytes conditions neurons against oxidative insult. J Neurosci 2004e;24:1101-1112. 